\title{
BMJ Open Effects of polymorphisms in APOA5 on the plasma levels of triglycerides and risk of coronary heart disease in Jilin, northeast China: a case-control study
}

\author{
Yueyue You, ${ }^{1}$ Yan-Hua Wu, ${ }^{2}$ Yangyu Zhang, ${ }^{1}$ Lili Zhang, ${ }^{1}$ Yan Song, ${ }^{1}$ Wei Bai, ${ }^{1}$ \\ Yuanyuan $\mathrm{Li},{ }^{1}$ Yaqin $\mathrm{Yu},{ }^{1}$ Changgui Kou ${ }^{1}$
}

To cite: You Y, Wu Y-H, Zhang Y, et al. Effects of polymorphisms in APOA5 on the plasma levels of triglycerides and risk of coronary heart disease in Jilin, northeast China: a case-control study. BMJ Open 2018;8:e020016. doi:10.1136/ bmjopen-2017-020016

\section{- Prepublication history for} this paper is available online. To view these files, please visit the journal online (http://dx.doi. org/10.1136/bmjopen-2017020016).

Received 10 0ctober 2017 Revised 24 March 2018 Accepted 3 May 2018

\section{Check for updates}

${ }^{1}$ Department of Epidemiology and Biostatistics, School of Public Health, Jilin University, Changchun, China

${ }^{2}$ Division of Clinical

Epidemiology, First Hospital of Jilin University, Changchun, China

Correspondence to

Dr Changgui Kou;

koucg@jlu.edu.cn

\section{ABSTRACT}

Objective The goal of this study is to investigate the associations of apolipoprotein A5 (APOA5) polymorphisms with coronary artery disease (CAD) in a Chinese population.

Method This case-control study included 710 subjects (355 patients with CAD and 355 controls) who were recruited from a cross-sectional study. Three single nucleotide polymorphisms (SNPs) rs662799 $(-1131 \mathrm{~T}>\mathrm{C})$, rs651821 (-3A>G) and rs2075291 (G185C) in APOA5 were selected and genotyped using the matrixassisted laser desorption ioniasation time of flight mass spectrometry technology. The $\chi^{2}$ test and haplotype analysis were performed to analyse the associations between APOA5 SNPs and CAD using the SPSS V.22.0 software package and the online SNPStats program. Results APOA5 SNPs rs662799 and rs651821 exhibited significant differences in genotype and allele distributions between patients with CAD and control subjects. The SNP rs662799 was significantly correlated with an increased risk of CAD when a dominant model was considered. The SNP rs651821 was significantly correlated with an increased risk of CAD when a codominant model was considered. Moreover, the variant $C$ alleles of rs662799 and the variant $G$ alleles of the rs651821 polymorphism were significantly correlated with increased plasma triglyceride (TG) levels in the CAD group (all $\mathrm{p}<0.05$ ). Additionally, a mediating effect of $\mathrm{TG}$ on the associations between the APOA5 rs662799 and rs651821 polymorphisms and CAD was observed.

Conclusion Based on these data, variants of the APOA5 gene are associated with CAD susceptibility and may modulate plasma TG levels among a Chinese population.

\section{INTRODUCTION}

Apolipoprotein A5 (APOA5) is a newly discovered member of the APOA4/APOC3/APOA1 apolipoprotein cluster that is located on chromosome 11q23 and has emerged as a significant modulator of serum triglyceride (TG) concentration. ${ }^{1}$ Functional studies with transgenic mice have revealed that $A P O A 5$-overexpressing mice display lower TG levels than controls; conversely, APOA5-knockout mice

\section{Strengths and limitations of this study}

- This study is significant because it is the first to use a mediation model to examine whether triglyceride levels act as a mediator of the associations between apolipoprotein A5 polymorphisms and coronary artery disease.

- Each patient included in our study is from a household in the selected communities, indicating a good representation.

- The population size is limited; future studies are needed to validate our results.

exhibit higher TG concentrations, indicating that $A P O A 5$ plays an important role in plasma TG metabolism. ${ }^{1}$

According to epidemiological studies, increased TG concentrations are related to an increased risk of coronary artery disease (CAD), the most common cause of death among both women and men over the age of 50 years. $^{2}$ High TG levels in combination with low high-density lipoprotein cholesterol (HDL-C) levels account for twice as many cases of CAD as low HDL-C alone. ${ }^{3}$

In humans, previous studies have consistently reported associations between common variants of $A P O A 5$ and differences in plasma TG levels. ${ }^{4-6}$ Consistent with this finding, the $A P O A 5-1131 \mathrm{~T}>\mathrm{C}$, c. $-3 \mathrm{~A}>\mathrm{G}$ and c.56C $>\mathrm{G}$ variants exhibit very strong associations with elevated TG levels in different racial groups, including Chinese, ${ }^{7}$ African Americans, Caucasians ${ }^{8}$ and Japanese schoolchildren, ${ }^{6}$ as well as a higher relative risk of developing dyslipidaemia. The strong associations between common genetic variations in the $A P O A 5$ gene and hypertriglyceridaemia raised the possibility that they may be related to CAD. As shown in a recent epidemiological study, the $A P O A 5-1131 \mathrm{~T}>\mathrm{C}$, c. $-3 \mathrm{~A}>\mathrm{G}$ and c.56C $>\mathrm{G}$ variants are associated with an 
increased risk of $\mathrm{CAD}$, through their associations with hypertriglyceridaemia. ${ }^{4910}$ However, there was report of a lack of association between $A P O A 5-1131 \mathrm{~T}>\mathrm{C}$ and c. $56 \mathrm{C}>\mathrm{G}$ variants and $\mathrm{CAD}$ risk conflicts with these data. ${ }^{11}$ This discrepancy could be explained by the observation that the distribution of the polymorphism of interest appears to be quite different in distinct ethnic populations, and these genetic differences may contribute to the varying prevalence rates of CAD among ethnic groups. Moreover, no other studies have confirmed these findings, and no similar studies have examined a northeastern Chinese population. Jilin is a special province because of specific weather, geographical location and eating habits of the population; thus, the prevalence of many chronic diseases, including $\mathrm{CAD}$ and metabolic syndrome, is high. Since different populations have contrasting profiles of CAD susceptibility and lipid risk factors, explorations of potential variations in the association between APOA5 and $\mathrm{CAD}$ between different ethnicities are necessary. More importantly, the study has examined the association between the APOA5 rs2075291 SNP and CAD. Therefore, in the present study, we chose two common SNPs, rs662799 and rs651821, and SNP rs2075291, which has not been described in a northeastern Chinese population and performed a case-control study to evaluate (1) whether APOA5 polymorphisms rs662799, rs651821, rs2075291 and their haplotypes are associated with CAD in a northeastern Chinese population and (2) whether TG acted as a mediator of the associations of the APOA5 polymorphisms with $\mathrm{CAD}$ using a mediation model.

\section{MATERIALS AND METHODS}

\section{Patient and public involvement}

A multistage stratified cluster sampling process was used to select the participants from nine areas of Jilin Province in northeast China in a community-based survey conducted in 2012. More detailed information about the sampling process is presented in previous publications. ${ }^{12-14}$ All participants were voluntarily involved in our research, including finishing a self-designed questionnaire that was previously developed to record information through person-to-person interviews. All trained staffs were involved in the recruitment to and conduct of the study. After the survey, we will send the results of all the participants to their home.

\section{Sampling}

In our study, 355 Han Chinese individuals with CAD from this survey were designated as cases, and 355 subjects without $\mathrm{CAD}$ were randomly selected from this survey as healthy controls. The inclusion criteria for the case group were: (a) angiographically confirmed coronary artery disease with $\geq 50 \%$ occlusion of $\geq 1$ major coronary arteries; (b) a confirmed myocardial infarction (MI) according to the WHO criteria for symptoms, elevated enzyme levels, or electrocardiographic changes; (c) no diagnosis of liver, thyroid, pituitary or renal disease; and (d) no history of acute or chronic inflammatory diseases.

The inclusion criteria for the healthy subjects were no history of MI and no evidence of MI on an echocardiogram. Individuals with any history or diagnoses of vascular disease, cancer, liver disease, renal disease, thyroid disease or acute or chronic inflammatory disease were excluded.

All subjects included in the present study signed a written informed consent form. Our study was approved by the Ethics Committee of the School of Public Health of Jilin University.

\section{Biochemical measurements}

Information about the demographic parameters age, gender, height, weight, alcohol consumption and smoking status was obtained from a questionnaire and the data for the body mass index (BMI), waist circumference, hip circumference, systolic blood pressure (SBP), diastolic blood pressure (DBP) and heart rate were measured in a physical examination. Alcohol consumption was categorised into three levels according self-reported drinking habits in number of times per week: normal drinker, frequent drinkers and never drinker. Subjects who drank more than three times per week were defined as frequent drinkers, subjects who drank more than one time but less than three times per week were defined as normal drinkers, and subjects who drank less than one time per week during their lifetimes were defined as never drinkers. The smoking status was categorised into current smoker, former smoker and never smoked, according to the self-reported number of cigarettes smoked and smoking days in the participants' lifetimes. Individuals who smoked at least 100 cigarettes in their lifetime and continued to smoke during the time of the survey were defined as current smokers, individuals who smoked at least 100 cigarettes but gave up smoking before the time of the survey were defined as former smokers and individuals who smoked $<100$ cigarettes during their lifetime were defined as never smokers. Systolic and diastolic blood pressure values were recorded by collecting an average of two measurements from each subjects while in a seated position after a 10 min rest. All subjects were asked to provide $5 \mathrm{~mL}$ of blood for biochemical analyses. The laboratory examinations included measurements of the levels of triglycerides (TG), total cholesterol (TC), low-density lipoprotein (LDL) and high-density lipoprotein (HDL). Blood samples were collected in the morning after an overnight fast, transported to the same laboratory under refrigeration and then stored at $-20^{\circ} \mathrm{C}$.

\section{SNP selection}

As described in previous studies, three SNPs rs2075291, rs662799 and rs651821 were selected by the Haploview program (http://hapmap.ncbi.nlm.nih.gov/) to identify the association between $A P O A 5$ gene polymorphism and $\mathrm{CAD}$. The minor allele frequencies of these three SNPs were $>0.05$ in the Chinese Han population. 


\section{DNA extraction and genotyping rate}

Genomic DNA was extracted from peripheral blood lymphocytes using a commercially available DNA isolation kit (Hangzhou, China). SNP genotyping was performed by matrix-assisted laser desorption ioniasation time of flight mass spectrometry (MALDI-TOF-MS) (Sequenom, San, Diego, California, USA) using the MassARRAY system. The detection rates for rs662799, rs651821 and rs2075291 were $99.4 \%$ (706/710), 99.7\% (708/710) and $99.0 \%(703 / 710)$, respectively.

\section{Statistical analysis}

The analyses were performed using the SPSS software (V.22.0). The Hardy-Weinberg equilibrium (HWE) was examined with the online SNPStats program (http:// bioinfo.iconcologia.net/SNPstats). Differences in the baseline characteristics between patients with $\mathrm{CAD}$ and controls were examined using Student's t-tests or $\chi^{2}$ tests. Differences in genotype between the CAD and control groups were examined using $\chi^{2}$ tests. The associations of $\mathrm{CAD}$ with the SNPs were examined using multivariate logistic regression analyses after adjusting for age, gender, BMI, smoking status, drinking, SBP and DBP. Pearson's $\chi^{2}$ tests were applied to compare the allele frequency of each polymorphism between the cases and controls. Plasma lipid levels in the CAD group were compared between the different genotypes by analysis of covariance. We selected the best models of inheritance for the SNPs based on the Akaike information criterion, and the haplotypes and associations were estimated using the SNPStats program. ${ }^{15}$

The mediation analysis was based on the model proposed by Baron et al. ${ }^{16}$ The following three multivariate linear regression models were examined, all of which were adjusted for age and gender: (1) $Y=c X+p_{1}$ age $+q_{1}$ gender $+e_{1} ;$ (2) $M=a x e+$ $p_{2}$ age $+q_{2}$ gender $+e_{2}$ and (3) $Y=c_{r} X+b M+p_{3}$ age $+q_{3}$ gender $+e_{3}$. The statistical test of the mediation effect included the following steps: (3) the association between the independent variable and the dependent variable (coefficient $c$ ) was assessed; (2) the association between the independent variable and the potential mediator (the coefficient $a$ ) was assessed; (3) both the independent variable and the potential mediator were simultaneously entered as predictors of the dependent variable, and the coefficient $b$ was determined to establish the significance of the mediation effect; and (4) if the mediation effect was significant, the type of mediation effect was determined by calculating the coefficient $c$, which indicated either a full mediation effect ( $c_{r}$ not significant) or a partial mediation effect ( $c_{r}$ remained significant).

All $p$ values were two-tailed and $p$ values $<0.05$ were considered statistically significant.

\section{RESULTS}

\section{Characteristics of the CAD patients and controls}

The general characteristics of the 355 patients with $\mathrm{CAD}$ and the 355 controls are presented in table 1 . The mean ages were $55.96 \pm 8.51$ years in the case group and $53.90 \pm 7.51$ years in the control group. The frequencies

\begin{tabular}{|c|c|c|}
\hline Parameters & Case $(n=355)$ & Control( $n=355)$ \\
\hline Age (years) & $55.96 \pm 8.51$ & $53.90 \pm 7.51$ \\
\hline Male, n $(\%)^{\star *}$ & $118(33.20)$ & $181(51.00)$ \\
\hline \multicolumn{3}{|l|}{ BP } \\
\hline $\mathrm{SBP}(\mathrm{mm} \mathrm{Hg})^{\star}$ & $137.82 \pm 23.62$ & $118.96 \pm 13.62$ \\
\hline $\mathrm{DBP}(\mathrm{mm} \mathrm{Hg})^{\star}$ & $82.62 \pm 13.05$ & $73.93 \pm 8.56$ \\
\hline \multicolumn{3}{|l|}{ Smoking, $\mathrm{n}(\%)^{\star \star}$} \\
\hline Current & 89 (25.10) & $114(32.10)$ \\
\hline Former & 37 (10.40) & $23(6.50)$ \\
\hline Never & $229(64.50)$ & $218(61.40)$ \\
\hline Drinking, $\mathrm{n}(\%)^{*}$ & 68 (19.20) & $106(29.86)$ \\
\hline BMI $\left(\mathrm{kg} / \mathrm{m}^{2}\right)^{*}$ & $25.23 \pm 4.02$ & $21.91 \pm 2.74$ \\
\hline $\mathrm{TG}(\mathrm{mmol} / \mathrm{L})^{*}$ & $2.23 \pm 1.70$ & $1.05 \pm 0.49$ \\
\hline $\mathrm{TC}(\mathrm{mmol} / \mathrm{L})^{*}$ & $5.24 \pm 1.09$ & $4.68 \pm 0.93$ \\
\hline LDL-C $(\mathrm{mmol} / \mathrm{L})^{*}$ & $3.18 \pm 0.92$ & $2.77 \pm 0.78$ \\
\hline $\mathrm{HDL}-\mathrm{C}(\mathrm{mmol} / \mathrm{L})^{*}$ & $1.37 \pm 0.42$ & $1.52 \pm 0.24$ \\
\hline
\end{tabular}

Continuous variables are presented as mean $\pm \mathrm{SD}$, and $\mathrm{n}(\%)$ for frequency variables.

$P$ values were analysed using Student's t-test and $\chi^{2}$ test. ${ }^{*} \mathrm{p}<0.05 ;{ }^{* *} \mathrm{p}<0.001$.

BP, blood pressure; BMI, body mass index; DBP, diastolic blood pressure; HDL-C, high-density lipoprotein cholesterol; LDL-C, low-density lipoprotein cholesterol; SBP, systolic blood pressure; TC, total cholesterol; TG, triglyceride.

of smoking and drinking were imbalanced between the different groups. As expected, compared with controls, patients with $\mathrm{CAD}$ exhibited a higher incidence of conventional cardiovascular risk factors, including higher SBP, DBP, BMI, plasma total cholesterol, TG and LDL-C levels, but lower plasma HDL-C levels.

\section{Distribution of the allele frequencies of the SNPs in the control and CAD patient groups}

The distributions of three SNPs (rs662799, rs651821 and rs2075291) were in HWE in the entire population as well as separately in the cases and controls. The comparisons of the genotype distributions and allele frequencies of the polymorphisms in the $A P O A 5$ gene between the subjects with and without $\mathrm{CAD}$ are presented in table 2. Two SNPs rs662799 and rs651821 exhibited significant differences in genotype distributions between the controls and patients with CAD ( $\mathrm{p}<0.05$ for all comparisons). Moreover, significant differences in allele distributions were observed between the controls and patients with CAD. However, differences in the genotype and allele frequency of SNP rs2075291 were not observed between the $\mathrm{CAD}$ and control groups.

\section{Genotype distribution comparison}

Table 3 presents the results of the multivariate logistic regression analysis employing the best selected model after adjusting for the confounding factors of age, gender, BMI, smoking status, drinking, systolic blood pressure and diastolic blood pressure. CAD was significantly associated with SNPs rs662799 and rs651821. In the dominant 


\begin{tabular}{|c|c|c|c|c|c|c|}
\hline \multirow{2}{*}{$\begin{array}{l}\text { SNPs } \\
\text { rs2075291 }\end{array}$} & \multicolumn{2}{|c|}{ Genotype/Allele } & \multirow{2}{*}{$\begin{array}{l}\text { Case(\%) } \\
167(47.00)\end{array}$} & \multirow{2}{*}{$\begin{array}{l}\text { Control(\%) } \\
198(55.80)\end{array}$} & \multirow{2}{*}{$\frac{\chi^{2}}{5.131}$} & \multirow{2}{*}{$\begin{array}{c}\text { P values } \\
0.077\end{array}$} \\
\hline & Genotype & GG & & & & \\
\hline & & $\mathrm{GC}$ & $100(28.20)$ & $81(22.80)$ & & \\
\hline & & $\mathrm{CC}$ & $83(23.40)$ & $74(20.80)$ & & \\
\hline & Allele & $\mathrm{G}$ & $434(61.13)$ & 477 (67.56) & 2.344 & 0.872 \\
\hline & & C & $266(38.87)$ & 229 (32.44) & & \\
\hline \multirow[t]{5}{*}{ rs662799 } & Genotype & $\pi$ & $123(34.70)$ & 179 (50.90) & 21.598 & $<0.001$ \\
\hline & & TC & $149(42.10)$ & $97(27.60)$ & & \\
\hline & & $\mathrm{CC}$ & $82(23.20)$ & 76 (21.60) & & \\
\hline & Allele & $\mathrm{T}$ & 395 (55.79) & 455 (64.63) & 11.512 & $<0.001$ \\
\hline & & $\mathrm{C}$ & $313(44.21)$ & 249 (35.37) & & \\
\hline \multirow[t]{5}{*}{ rs651821 } & Genotype & AA & $137(38.70)$ & $188(53.10)$ & 17.450 & $<0.001$ \\
\hline & & $A G$ & $130(36.70)$ & $113(31.90)$ & & \\
\hline & & GG & $87(24.60)$ & $53(15.00)$ & & \\
\hline & Allele & $A$ & $404(57.06)$ & $489(69.07)$ & 21.905 & $<0.001$ \\
\hline & & $\mathrm{G}$ & 304 (42.94) & 219 (30.933) & & \\
\hline
\end{tabular}

CAD, coronary artery disease.

model, we observed a significant association between CAD risk and the SNP rs662799. In the codominant model, we also observed an association between CAD risk and SNP rs2075291.

\section{APOA5 haplotype analysis}

We performed a haplotype analysis to determine whether the three SNPs in the APOA5 gene were associated with CAD. Three haplotypes were identified in the APOA5 gene in our population. As shown in table 4, two haplotypes were associated with an increased risk of CAD (ORs=1.64 and 2.19).

\section{APOA5 SNPs rs2075291, rs662799 and rs651821 and the plasma lipid profile}

Table 5 presents the mean plasma lipid levels among the genotypes of APOA5 SNPs rs2075291, rs662799 and rs651821 in the CAD groups. As expected, the APOA5 rs662799 $\mathrm{C}$ alleles and rs651821 G allele significantly correlated with increased plasma TG levels in the CAD group (all $\mathrm{p}<0.05)$. However, no other significant differences were detected between the $A P O A 5$ polymorphism in SNP rs2075291 and plasma TG levels.

\section{Mediation analysis of the APOA5 rs662799 and rs651821 polymorphisms, TG and CAD}

We used a mediation model to examine whether TG levels acted as a mediator of the associations of the APOA5 rs651821 and rs662799 polymorphisms with CAD as illustrated in figure $1 \mathrm{~A}$. Here, 'a', 'b', 'c' and 'c ' are used to represent the coefficients in the mediation analysis model. The number of APOA5 rs651821 $\mathrm{G}$ alleles positively correlated with CAD (c=0.9029, $\mathrm{p}<0.001)$. A positive correlation was observed between the number of the APOA5 rs651821 G alleles and TG levels $(\mathrm{a}=0.4408$, $\mathrm{p}<0.05)$. By testing ' $\mathrm{b}$ ', we observed a significant mediation effect $(b=1.6916, p<0.05)$, and the insignificance

\begin{tabular}{|c|c|c|c|c|c|c|}
\hline SNPs & Model & Genotype & Case (\%) & Control (\%) & OR $(95 \% \mathrm{Cl})^{*}$ & $P$ values \\
\hline \multirow[t]{2}{*}{ rs2075291 } & Recessive & $\mathrm{G} / \mathrm{G}-\mathrm{G} / \mathrm{C}$ & 267 (76.3) & $279(79.0)$ & 1.00 & 0.300 \\
\hline & & $\mathrm{C} / \mathrm{C}$ & $83(23.7)$ & $74(21.0)$ & 1.22 (0.84 to $1.76 \rrbracket$ & \\
\hline \multirow[t]{2}{*}{ rs662799 } & Dominant & $T / T$ & $123(34.8)$ & $179(50.9)$ & 1.00 & $<0.001$ \\
\hline & & $\mathrm{T} / \mathrm{C}-\mathrm{C} / \mathrm{C}$ & 231 (65.2) & $173(49.1)$ & 1.61 (1.09 to $2.37 区$ & \\
\hline \multirow[t]{3}{*}{ rs651821 } & Codominant & $\mathrm{A} / \mathrm{A}$ & $137(38.7)$ & $188(53.1)$ & 1.00 & $<0.001$ \\
\hline & & $\mathrm{G} / \mathrm{A}$ & $130(36.7)$ & $113(31.9)$ & 1.49 (1.04 to 2.16$)$ & \\
\hline & & $\mathrm{G} / \mathrm{G}$ & 87 (24.6) & $53(15.0)$ & 2.23 (1.43 to 3.47$)$ & \\
\hline
\end{tabular}

${ }^{*}$ Adjusted for age, gender, BMI, smoking status, drinking, systolic blood pressure and diastolic blood pressure.

$\mathrm{BMI}$, body mass index; CAD, coronary artery disease; SNP, single nucleotide polymorphism. 
Table 4 Association analysis of haplotypes derived from polymorphic sites using genotype data

\begin{tabular}{|c|c|c|c|c|c|c|c|c|}
\hline \multirow[b]{2}{*}{ Haplotype } & \multicolumn{3}{|l|}{ SNP } & \multicolumn{3}{|c|}{ Frequency } & \multirow[b]{2}{*}{ Adjusted OR $(95 \% \mathrm{Cl})^{*}$} & \multirow[b]{2}{*}{$P$ values } \\
\hline & rs651821 & rs662799 & rs2075291 & Total & Cases & Controls & & \\
\hline 1 & $A$ & C & $G$ & 0.7169 & 0.7505 & 0.6636 & 1.00 & - \\
\hline 3 & G & $\mathrm{T}$ & $\mathrm{T}$ & 0.0596 & 0.0509 & 0.0733 & $2.19(1.22-3.95)$ & 0.009 \\
\hline Rare† & * & * & * & 0.0065 & 0.0023 & 0.0038 & $0.33(0.05-2.40)$ & 0.280 \\
\hline
\end{tabular}

*OR was adjusted for age, gender, BMI, smoking status, drinking, systolic blood pressure and diastolic blood pressure.

†Haplotypes with frequencies $<0.01$.

BMI, body mass index; SNP, single nucleotide polymorphism.

of ' $c_{r}$ ' indicated a partial mediation effect $\left(c_{r}=0.1553\right.$, $\mathrm{p}<0.05)$. Further calculations $(\mathrm{ab} / \mathrm{c} \times 100 \%)$ revealed a mediation effect of $82.58 \%$. Moreover, similar results were obtained in the mediation analysis; TG levels exerted a partial mediation effect on the association between the APOA5 rs662799 polymorphism and CAD (figure 1B); the mediation effect was $(\mathrm{ab} / \mathrm{c} \times 100 \%) 46.60 \%$.

\section{DISCUSSION}

Our case-control study including Chinese subjects revealed that SNPs rs662799 and rs651821 were significantly associated with an increased risk of CAD that was accompanied by elevated plasma TG levels after adjusting for age, gender, BMI, smoking status, drinking status, SBP and DBP. The $\mathrm{C}$ allele frequency of APOA5 rs662799 (30\%) observed here was similar to frequencies observed in previously studies of Japanese ${ }^{17}$ and Chinese subjects $^{7} 1819$ but was much higher than the frequency reported for Caucasian populations $(10 \%)$.

The association observed in our study between the $A P O A 5$ rs662799 variant and the increased risk of CAD is consistent with previous reports examining Korean ${ }^{20}$ and Czech populations. ${ }^{21} \mathrm{~A}$ recent study also reported similar results ${ }^{22}$; Szalai et al reported a significantly higher frequency of the APOA5 $\mathrm{C}$ allele among patients with CAD than among controls $(\mathrm{OR}=1.98 ; 95 \% \mathrm{CI} 1.14$ to 3.48; $\mathrm{p}<0.001)$. However, other studies have not identified similar associations. ${ }^{23}{ }^{24}$ In the present study, the APOA5 rs651821 polymorphism was a risk factor for CAD among a Chinese population. Our results are not consistent with the results of previous studies that have supported the hypothesis that rs651821 is not significantly associated with CAD risk in a Chinese population. ${ }^{25-27}$ This discrepancy might be explained by the fact that our study was a case-control study, and the cases were limited to survivors of CAD. This difference would certainly affect the full influence of the examined polymorphisms.

Some studies have reported that $A P O A 5$ polymorphisms are associated with lipid levels among diverse ethnic groups. ${ }^{1528-30}$ We also successfully confirmed this association in the present study. For both APOA5 rs662799 and rs651821, patients with CAD presenting with the CC and

Table 5 Association between APOA5 genotype variants and clinical and biochemical parameters in CAD group

\begin{tabular}{|c|c|c|c|c|c|}
\hline \multirow[b]{2}{*}{ SNP } & \multirow[b]{2}{*}{ Genotype } & \multicolumn{4}{|l|}{ Parameters } \\
\hline & & $\mathrm{TG}(\mathrm{mmol} / \mathrm{L})$ & TC (mmol/L) & LDL-C (mmol/L) & HDL-C (mmol/L) \\
\hline \multirow[t]{4}{*}{ rs2075291 } & $\mathrm{GG}(n=135)$ & $2.302 \pm 1.785$ & $5.229 \pm 1.076$ & $3.087 \pm 0.847$ & $1.395 \pm 0.504$ \\
\hline & $\mathrm{GC}(n=132)$ & $2.640 \pm 1.958$ & $5.204 \pm 1.167$ & $3.230 \pm 1.077$ & $1.181 \pm 0.295$ \\
\hline & $\mathrm{CC}(\mathrm{n}=83)$ & $1.719 \pm 1.043$ & $5.334 \pm 1.054$ & $3.371 \pm 0.930$ & $1.486 \pm 0.209$ \\
\hline & $P$ values & 0.052 & 0.705 & 0.058 & 0.056 \\
\hline \multirow[t]{4}{*}{ rs662799 } & $T T(n=123)$ & $1.900 \pm 1.339$ & $5.228 \pm 1.012$ & $3.226 \pm 0.904$ & $1.439 \pm 0.416$ \\
\hline & TC $(n=100)$ & $2.507 \pm 1.913$ & $5.274 \pm 1.187$ & $3.165 \pm 0.939$ & $1.297 \pm 0.403$ \\
\hline & $C C(n=131)$ & $2.811 \pm 2.121$ & $5.125 \pm 1.149$ & $3.009 \pm 0.982$ & $1.266 \pm 0.440$ \\
\hline & $\mathrm{P}$ values & 0.010 & 0.764 & 0.428 & 0.403 \\
\hline \multirow[t]{4}{*}{ rs651821 } & AA $(n=137)$ & $1.914 \pm 1.352$ & $5.221 \pm 1.017$ & $3.215 \pm 0.904$ & $1.441 \pm 0.416$ \\
\hline & $A G(n=130)$ & $2.496 \pm 1.913$ & $5.295 \pm 1.181$ & $3.182 \pm 0.936$ & $1.303 \pm 0.412$ \\
\hline & $\mathrm{GG}(\mathrm{n}=87)$ & $2.884 \pm 2.110$ & $5.071 \pm 1.156$ & $2.971 \pm 0.992$ & $1.214 \pm 0.382$ \\
\hline & $P$ values & 0.001 & 0.543 & 0.363 & 0.401 \\
\hline
\end{tabular}

$P$ values were calculated by ANCOVA.

ANCOVA, analysis of variance; CAD, coronary artery disease; HDL-C, high-density lipoprotein cholesterol; LDL-C, low-density lipoprotein cholesterol; TC, total cholesterol; TG, triglyceride. 


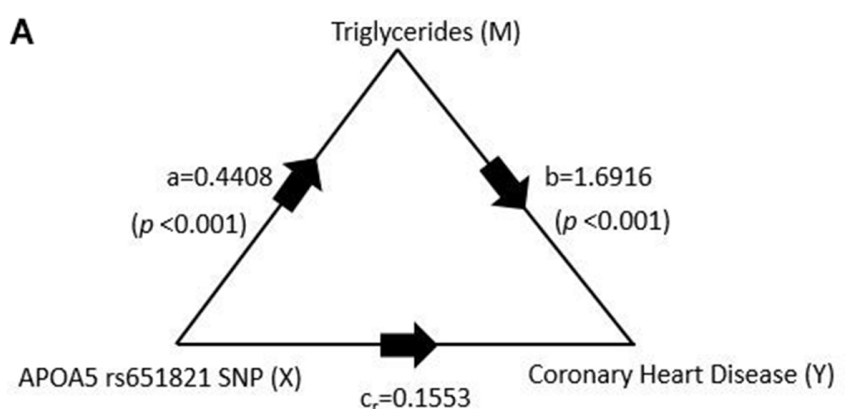

$(p<0.001)$

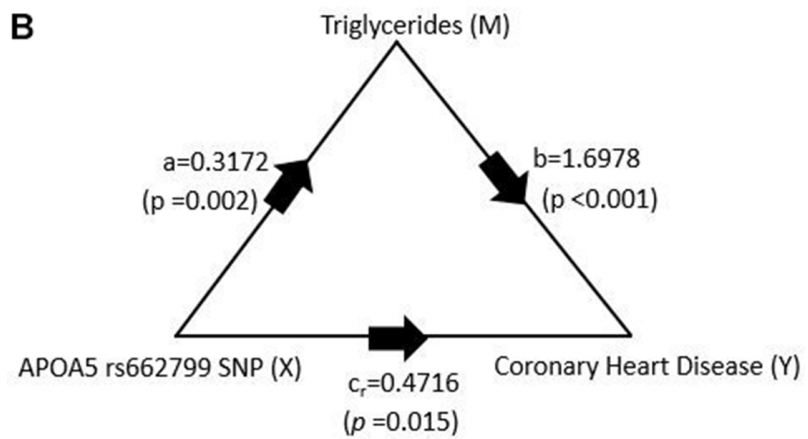

Figure 1 Mediation analysis APOA5 rs651821 and rs662799 SNP, TGs and CAD. In order to distinguish among the three linear regression models constructed in the mediation analysis, the coefficients in the model were represented by ' $a$,' $b$,' ' $c$ ' and ' $c_{r}$ '. The first regression model used ' $c$ ' as the coefficient of SNP in association with CAD; the second model used ' $a$ ' as the coefficient of SNP in association with TGs; the third model put TGs and SNP as independent variables simultaneously, and used ' $b$ ' and ' $\mathrm{C}_{r}$ ' as the coefficients in association with CAD. ' $\mathrm{X}$ ', ' $\mathrm{Y}$ ' and ' $M$ ' were used to represent the independent variable, the dependent variable and the mediator in the mediation analysis model. APOA5, apolipoprotein A5; CAD, coronary heart disease; SNP, single nucleotide polymorphism; TG, triglyceride.

GG genotypes exhibited higher TG levels than subjects with the TT and AA genotypes, respectively. However, we did not observe a significant association between the APOA5 rs2075291 SNP and plasma TG levels. Similar to previous reports, the TG levels were approximately $25 \%$ higher in carriers of the APOA5 rs662799 $\mathrm{C}$ allele than in non-carriers, indicating that this allele is also an important genetic determinant of triglyceride levels in our Chinese population.

Researchers have not clearly determined whether the increased risk of CAD is due to the function of APOA5 per se or mediated by changes in the atherogenic lipid profile. Hence, we used a mediation model to examine whether TG levels acted as a mediator of the associations of the APOA5 rs651821 and rs662799 polymorphisms with CAD. TG levels were a significant mediator in this model. The mediation model was used for the first time to analyse the associations between $A P O A 5$ polymorphisms, TG levels and CAD. Our study identified a novel mechanism underlying the established association between
APOA5 polymorphisms and CAD in large epidemiological studies. Based on accumulating evidence, high TG levels and genetic variations are associated with increased cardiovascular morbidity and mortality risks. ${ }^{31}$ However, many determinants have not yet been clarified because genetic variation explains only $5 \%-50 \%$ of the overall variations in TG levels and CAD risk. ${ }^{32}$ Environmental and lifestyle variations may affect the observed associations between the genetic variants and disease outcomes.

Our study has certain limitations. First, glycaemia is one of the most important CV risk factors; thus, diabetes should have been included in our study. However, because of limited funding, we were unable to test for diabetes and plasma APOA5 levels; we plan to perform a further study in the future when we have sufficient funding. Second, our study was a case-control study; thus, the cause-effect relationships between the APOA5 rs662799 and rs651821 genotypes and TG and HDL-C concentrations were not easy to establish. In addition, CAD is a complex disease and several SNPs have been reported to correlate with $\mathrm{CAD}$ and myocardial ischaemia in other populations. ${ }^{33-37}$ We did not study these SNPs because of limited funding. $\mathrm{CAD}$ is a complex disease with a complex pathophysiology caused by interactions between multiple endogenous and exogenous factors. Many diagnostic criteria for CAD have been reported. In our study, all healthy subjects had no history of MI and no evidence of MI on an echocardiogram, but this method may not exclude potential patients with $\mathrm{CAD}$ who are asymptomatic and did not experience an infarction. Therefore, in future studies, subjects without CAD should be chosen using stricter criteria. Despite these limitations, our study confirmed that common variants of the APOA5 gene are associated with $\mathrm{CAD}$ and contribute to the variation in human plasma TG levels. Therefore, $A P O A 5$ variants are significant predictors of a high TG risk. Variations in this gene may be associated with the development of CAD. As reported in the recent study by Caussy et $a l .{ }^{38}$ miRNAs may be involved in the association of $A P O A 5$ with $\mathrm{CAD}$; therefore, we plan to perform additional studies to confirm our hypothesis of the functions of these SNPs and validate our findings.

\section{CONCLUSION}

In conclusion, APOA5 SNPs rs662799 and rs651821 were significantly associated with an increased risk of CAD and accompanied by elevated plasma TG levels. According to the results of the mediation analysis, the effects of rs662799 and rs651821 on CAD were mediated by TG levels.

Acknowledgements The authors wish to thank the study participants for their contribution to the research, as well as current and past investigators and staff. They would like to express their sincere gratitude and deep appreciation to all patient advisers.

Contributors YY, Y-HW, YZ, LZ, CK and YY: designed and performed the study. YY, Yan-HuaWu, YZ and YS: analysed the data. YY: drafted the manuscript. YY, WB, YL and $Y Z$ : participated in revising draft of the manuscript. All authors approved the final version of the manuscript. 
Funding This study was funded by The scientific research foundation of the Jilin Provincial Health Department, China (grant\#2011Z116).

Competing interests None declared.

Patient consent Obtained.

Ethics approval All participants in this study provided written informed consent form and our study was approved by the ethics committee of the School of Public Health, Jilin University (reference number: 2012-R-011).

Provenance and peer review Not commissioned; externally peer reviewed.

Data sharing statement No additional data are available.

Open Access This is an Open Access article distributed in accordance with the Creative Commons Attribution Non Commercial (CC BY-NC 4.0) license, which permits others to distribute, remix, adapt, build upon this work non-commercially, and license their derivative works on different terms, provided the original work is properly cited and the use is non-commercial. See: http://creativecommons.org/ licenses/by-nc/4.0/

(c) Article author(s) (or their employer(s) unless otherwise stated in the text of the article) 2018. All rights reserved. No commercial use is permitted unless otherwise expressly granted.

\section{REFERENCES}

1. Pennacchio LA, Olivier M, Hubacek JA, et al. An apolipoprotein influencing triglycerides in humans and mice revealed by comparative sequencing. Science 2001;294:169-73.

2. De Andrade FM, Maluf SW, Schuch JB, et al. The influence of the S19W SNP of the APOA5 gene on triglyceride levels in southern Brazil: interactions with the APOE gene, sex and menopause status. Nutr Metab Cardiovasc Dis 2011;21:584-90.

3. Castelli WP. Epidemiology of triglycerides: a view from Framingham. Am J Cardiol 1992;70:H3-9.

4. Pennacchio LA, Olivier M, Hubacek JA, Krauss RM, Rubin EM, Cohen JC. Two independent apolipoprotein A5 haplotypes influence human plasma triglyceride levels. Hum Mol Genet 2002;11:3031-8.

5. Aouizerat BE, Kulkarni M, Heilbron D, et al. Genetic analysis of a polymorphism in the human apoA-V gene: effect on plasma lipids. $J$ Lipid Res 2003;44:1167-73.

6. Endo K, Yanagi H, Araki J, et al. Association found between the promoter region polymorphism in the apolipoprotein $\mathrm{A}-\mathrm{V}$ gene and the serum triglyceride level in Japanese schoolchildren. Hum Genet 2002;111:570-2.

7. Baum L, Tomlinson B, Thomas GN. APOA5-1131T>C polymorphism is associated with triglyceride levels in Chinese men. Clin Genet 2003;63:377-9.

8. Pennacchio LA, Olivier M, Hubacek JA, et al. Two independent apolipoprotein A5 haplotypes influence human plasma triglyceride levels. Hum Mol Genet 2002;11:3031-8.

9. Sarwar N, Sandhu MS, Ricketts SL, et al. Triglyceride-mediated pathways and coronary disease: collaborative analysis of 101 studies. Lancet 2010;375:1634-9.

10. Talmud PJ, Hawe E, Martin S, et al. Relative contribution of variation within the APOC3/A4/A5 gene cluster in determining plasma triglycerides. Hum Mol Genet 2002;11:3039-46.

11. Lee KW, Ayyobi AF, Frohlich JJ, et al. APOA5 gene polymorphism modulates levels of triglyceride, HDL cholesterol and FERHDL but is not a risk factor for coronary artery disease. Atherosclerosis 2004;176:165-72.

12. Wang S, Kou C, Liu Y, et al. Rural-urban differences in the prevalence of chronic disease in northeast China. Asia Pac J Public Health 2015;27:394-406.

13. Wang SB, D'Arcy C, Yu YQ, et al. Prevalence and patterns of multimorbidity in northeastern China: a cross-sectional study. Public Health 2015;129:1539-46.

14. Wang R, Zhang P, Gao C, et al. Prevalence of overweight and obesity and some associated factors among adult residents of northeast China: a cross-sectional study. BMJ Open 2016;6:e010828.

15. Solé X, Guinó E, Valls J, et al. SNPStats: a web tool for the analysis of association studies. Bioinformatics 2006;22:1928-9.

16. Baron RM, Kenny DA. The moderator-mediator variable distinction in social psychological research: conceptual, strategic, and statistical considerations. J Pers Soc Psychol 1986;51:1173-82.
17. Yamada $\mathrm{Y}$, Ando $\mathrm{F}$, Shimokata $\mathrm{H}$. Association of the genetic variants of APOA5 and PRKCH with hypertension in community-dwelling Japanese individuals. Mol Med Rep 2008;1:407-14.

18. Lai CQ, Tai ES, Tan CE, et al. The APOA5 locus is a strong determinant of plasma triglyceride concentrations across ethnic groups in Singapore. J Lipid Res 2003;44:2365-73.

19. Li GP, Wang JY, Yan SK, et al. Genetic effect of two polymorphisms in the apolipoprotein A5 gene and apolipoprotein C3 gene on serum lipids and lipoproteins levels in a Chinese population. Clin Genet 2004;65:470-6.

20. Jang Y, Paik JK, Hyun YJ, et al. The apolipoprotein A5 -1131T>C promoter polymorphism in Koreans: association with plasma APOA5 and serum triglyceride concentrations, LDL particle size and coronary artery disease. Clin Chim Acta 2009;402:83-7.

21. Hubacek JA, Skodova Z, Adamkova V, et al. Apolipoprotein AV gene polymorphisms (T-1131/C and Ser19/Trp) influence plasma triglyceride levels and risk of myocardial infarction. Exp Clin Cardiol 2003;8:126.

22. Szalai C, Keszei M, Duba J, et al. Polymorphism in the promoter region of the apolipoprotein A5 gene is associated with an increased susceptibility for coronary artery disease. Atherosclerosis 2004;173:109-14.

23. Talmud PJ, Martin S, Taskinen MR, et al. APOA5 gene variants, lipoprotein particle distribution, and progression of coronary heart disease: results from the LOCAT study. J Lipid Res 2004;45:750.

24. Wong WM, Hawe E, Li LK, et al. Apolipoprotein AIV gene variant S347 is associated with increased risk of coronary heart disease and lower plasma apolipoprotein AIV levels. Circ Res 2003;92:969-75.

25. Fu Q, Tang X, Chen J, et al. Effects of Polymorphisms in APOA4APOA5-ZNF259-BUD13 Gene Cluster on Plasma Levels of Triglycerides and Risk of Coronary Heart Disease in a Chinese Han Population. PLoS One 2015;10:e0138652.

26. Ken-Dror G, Goldbourt U, Dankner R. Different effects of apolipoprotein A5 SNPs and haplotypes on triglyceride concentration in three ethnic origins. J Hum Genet 2010;55:300-7.

27. Ou HJ, Huang G, Liu W, et al. Relationship of the APOA5/A4/C3/ A1 gene cluster and APOB gene polymorphisms with dyslipidemia. Genet Mol Res 2015;14:9277-90.

28. Austin MA, Talmud PJ, Farin FM, et al. Association of apolipoprotein A5 variants with LDL particle size and triglyceride in Japanese Americans. Biochim Biophys Acta 2004;1688:1-9.

29. Kuulasmaa K, Tunstall-Pedoe H, Dobson A, et al. Estimation of contribution of changes in classic risk factors to trends in coronaryevent rates across the WHO MONICA Project populations. The Lancet 2000;355:675-87.

30. Jang Y, Kim JY, Kim OY, et al. The -1131T-->C polymorphism in the apolipoprotein A5 gene is associated with postprandial hypertriacylglycerolemia; elevated small, dense LDL concentrations; and oxidative stress in nonobese Korean men. Am J Clin Nutr 2004;80:832-40.

31. Kathiresan S, Willer CJ, Peloso GM, et al. Common variants at 30 loci contribute to polygenic dyslipidemia. Nat Genet 2009;41:56-65.

32. Kathiresan S, Melander O, Anevski D, et al. Polymorphisms associated with cholesterol and risk of cardiovascular events. $N$ Engl J Med 2008;358:1240-9.

33. Frikke-Schmidt R, Nordestgaard BG, Jensen GB, et al. Genetic variation in ABCA1 predicts ischemic heart disease in the general population. Arterioscler Thromb Vasc Biol 2008;28:180-6.

34. Mosley JD, van Driest SL, Wells QS, et al. Defining a Contemporary Ischemic Heart Disease Genetic Risk Profile Using Historical Data. Circ Cardiovasc Genet 2016:9:521-30.

35. Fedele F, Mancone M, Chilian WM, et al. Role of genetic polymorphisms of ion channels in the pathophysiology of coronary microvascular dysfunction and ischemic heart disease. Basic Res Cardiol 2013;108:387.

36. Stengård JH, Frikke-Schmidt R, Tybjaerg-Hansen A, et al. Variation in 5 ' promoter region of the APOE gene contributes to predicting ischemic heart disease (IHD) in the population at large: the Copenhagen City Heart Study. Ann Hum Genet 2007;71:762-71.

37. Vecoli C, Adlerstein D, Shehi E, et al. Genetic score based on high-risk genetic polymorphisms and early onset of ischemic heart disease in an Italian cohort of ischemic patients. Thromb Res 2014;133:804-10.

38. Caussy $C$, Charrière $S$, Marçais $C$, et al. An APOA5 3' UTR variant associated with plasma triglycerides triggers APOA5 downregulation by creating a functional miR-485-5p binding site. Am J Hum Genet 2014;94:129-34. 